\title{
Genetic Variability in Ridge Gourd (Luffa acuntangula (L.) Roxb.)
}

\author{
K. Akhila* and Devi Singh \\ Department of Horticulture, Naini Agricultural Institute, Sam Higginbottom University of \\ Agriculture, Technology and Sciences, Prayagraj-211007, India \\ *Corresponding author
}

\section{A B S T R A C T}

\section{Keywords}

Ridge gourd, Genotypes, Genetic variability,

Heritability

Article Info

Accepted:

20 September 2020

Available Online:

10 October 2020

\begin{abstract}
An experiment was conducted on Genetic variability in the eighteen genotypes of Ridge Gourd during 2019-20 at the Research Field of Department of Horticulture, Sam Higginbottom University of Agriculture, Technology and Sciences, Prayagraj. The observations were recorded on various yield and yield contributing characters. The results from the present investigation revealed that On the basis of Based on mean performance for fruit yield per plant $(2.720 \mathrm{~kg})$ and fruit yield $\left(\left(24.480 \mathrm{t} / \mathrm{ha}^{-1}\right)\right.$, genotypes 2017/RIGHYB-5 were considered suitable genotypes in Prayagraj climatic condition. Coefficient of variation revealed that high magnitude of GCV and PCV were recorded for Fruit yield/ ha (ton) and Average fruit weight (g). The heritability estimates were found to be high (more than 60\%). The genetic advance and genetic advance as percent of mean estimates were found to be high (more than 20\%). Genotypic correlation coefficient analysis revealed that fruit yield plant $^{-1}(\mathrm{~kg})$ showed positive significant association with Fruit length $(\mathrm{cm})\left(0.598^{* *}\right)$, Fruit diameter $(\mathrm{cm})\left(0.741^{* *}\right)$, Rind thickness $(\mathrm{mm})$ $\left(0.514^{* *}\right)$, Flash thickness $(\mathrm{mm})\left(0.523^{* *}\right)$, Number of fruit per plant $\left(0.666^{* *}\right)$, Vine length $(\mathrm{cm})$ at 90 DAS $\left(0.275^{*}\right)$ and Average fruit weight $(\mathrm{g})\left(0.944^{* *}\right)$ at genotypic level. Whereas Phenotypic correlation coefficient analysis revealed that fruit yield plant $^{-1}(\mathrm{~kg})$ showed positive significant association with Fruit length $(\mathrm{cm})\left(0.573^{* *}\right)$, Fruit diameter (cm) $(0.709 * *)$, Rind thickness $(\mathrm{mm})(0.509 * *)$, Flash thickness $(\mathrm{mm})(0.504 * *)$, Number of fruit per plant $(0.607 * *)$, Average fruit weight $(\mathrm{g})(0.924 * *)$ and Vine length $(\mathrm{cm})$ at 90 DAS $\left(0.270^{*}\right)$ at phenotypic level.
\end{abstract}

\section{Introduction}

Ridge gourd [Luffaacutangula (L.)Roxb.], popularly known as Kalitori and also called as angled gourd, angled loofah, Chinese okra, silky gourd and ribbed gourd, belongs to genus Luffa of "Cucurbitaceae" family and has chromosome number $2 \mathrm{n}=26$.
India has the credibility of producing 169.478 million tonnes of vegetables covering an area of 9.542 million hectares in 2014-15 (NHB, 2014-15) securing the second status among the vegetable producing countries of the world but the per capita availability of vegetables in India is as low as $160 \mathrm{gm}$ as against the recommended 300 g per day by FAO. 
Fruit contain moisture $92.5 \mathrm{~g}$, protein $0.5 \mathrm{~g}$, fat $0.5 \mathrm{~g}$, carbohydrate $3.4 \mathrm{~g}$, energy $17 \mathrm{k}$ cal, calcium $18 \mathrm{mg}$, vitamin C $5 \mathrm{mg}$, riboflavin $0.01 \mathrm{mg}$, phosphorous $26 \mathrm{mg}$, iron $0.5 \mathrm{mg}$ and carotene $33 \mu \mathrm{g}$ per $100 \mathrm{~g}$ of edible portion (Sheshadri and Parthasarthy, 1980). Besides their use as vegetables, it is also used for various purposes. The fiber obtained from the mature dry fruit is used in industry for filters of various sorts, good pot holders, table mats, bath room mats, slipper and shoe soles. The fiber is also proved to be a good insulator for various purposes. Sometimes the dry fruits which gave good storability are used for ornamental purposes also. It is emetic and traditionally used for the treatment of stomach ailment and fever (Chakravarthy, 1959).

The success of any crop improvement programme largely depends upon the nature and magnitude of genetic variability existing in the breeding material. This study on genetic variability and divergence elucidates information on genetic parameters. Further, path- coefficient technique provides the information on the direct and indirect contribution of individual characters towards yield. Based on these studies, the importance of individual character is marked to facilitate the selection programme for larger gains. Generally, diverse germplasm are expected to give high hybrid vigour. Hence, it necessitates studying the genetic divergence among the existing genotypes for identification of parents for hybridization programme.

\section{Materials and Methods}

The present research work entitled, "Genetic variability in Ridge Gourd (Luffa acuntangula (L.) Roxb.)" was conducted to study the variability, heritability, genetic advance, and correlation and their effect on yield and yield contributing traits. On 18 genotypes of Ridge Gourd collected from different institutes, research stations and private seed companies.

\section{Experimental site}

The study was conducted in the Research Field, Department of Horticulture, Naini Agriculture Institute, SHUATS, Prayagraj, located between $25.87^{\circ}$ North latitude $81.15^{\circ}$ East latitude. The altitude is 78 meters above the mean sea level.

\section{Soil type}

The soil type of experimental field was sandy loam with average fertility level and $\mathrm{pH}$ in the range of 7.0 to 8.0 .

\section{Climate}

Department of Horticulture, Naini Agricultural Institute, SHUATS, Prayagraj, falls under the humid subtropical zone. Maximum rainfall received during the period between July and the end of September. However, occasional showers are also very common in the month of June, December and January. The winter month will usually cool and dry. The summer is hot and dry western hot wind start from April and end at onset of monsoon.

\section{Results and Discussion}

Analysis of variance showed significant differences among the genotypes for the eighteen characters studied. Analysis of variance showed significant difference among the genotypes for the different characters at $0.1 \%$ and $5 \%$ significance.

Similar finding for variance have also been reported by Ramakant et al., (2008), Parameshwarappa et al., (2008), Kumar et al., (2012), Reddy et al., (2013), Rani and Kumar (2013) and Narayanan and Murugan (2013), Singh et al., 2015 and Paul et al., 2015 reported significant mean sum of square for various quantitative and quality traits in cow pea (Table 1-7). 
Table.1 List of genotypes were used in the present investigation

\begin{tabular}{|c|c|c|}
\hline Sl. No. & Genotype Symbol & Genotypes \\
\hline 1 & $\mathrm{G}_{1}$ & $2018 /$ RIG HYB-1 \\
\hline 2 & $\mathrm{G}_{2}$ & $2018 /$ RIG HYB-2 \\
\hline 3 & $\mathrm{G}_{3}$ & $2018 /$ RIG HYB-3 \\
\hline 4 & $\mathrm{G}_{4}$ & $2018 /$ RIG HYB-4 \\
\hline 5 & $\mathrm{G}_{5}$ & $2018 /$ RIG HYB-5 \\
\hline 6 & $\mathrm{G}_{6}$ & $2018 /$ RIG HYB-6 \\
\hline 7 & $\mathrm{G}_{7}$ & $2018 /$ RIG HYB-7 \\
\hline 8 & $\mathrm{G}_{8}$ & $2017 /$ RIG HYB-1 \\
\hline 9 & $\mathrm{G}_{9}$ & $2017 /$ RIG HYB-2 \\
\hline 10 & $\mathrm{G}_{10}$ & $2017 /$ RIG HYB-5 \\
\hline 11 & $\mathrm{G}_{11}$ & $2017 /$ RIG HYB-6 \\
\hline 12 & $\mathrm{G}_{12}$ & $2017 /$ RIG VAR-1 \\
\hline 13 & $\mathrm{G}_{13}$ & $2017 /$ RIG VAR-2 \\
\hline 14 & $\mathrm{G}_{14}$ & $2017 /$ RIG VAR-3 \\
\hline 15 & $\mathrm{G}_{15}$ & $2017 /$ RIG VAR-4 \\
\hline 16 & $\mathrm{G}_{16}$ & $2017 /$ RIG VAR-5 \\
\hline 17 & $\mathrm{G}_{17}$ & $2017 /$ RIG VAR-6 \\
\hline 18 & $\mathrm{G}_{18}$ & Rekha \\
\hline
\end{tabular}

Table.2 Analysis of variance for 18 characters in 18 genotypes of ridge gourd

\begin{tabular}{|l|l|c|c|c|}
\hline \multirow{2}{*}{ Sl.No. } & \multicolumn{1}{|c|}{ Character } & \multicolumn{3}{|c|}{ Mean sum of square } \\
\cline { 3 - 5 } & & $\begin{array}{c}\text { Replications } \\
\text { (df = 2) }\end{array}$ & $\begin{array}{c}\text { Treatments } \\
\text { (df = 17) }\end{array}$ & $\begin{array}{c}\text { Error } \\
\text { (df = 34) }\end{array}$ \\
\hline $\mathbf{1}$ & Vine Length (cm) at 90 DAS & 104.18 & 3956.21 & 69.81 \\
\hline $\mathbf{2}$ & Days to first female flower & 0.339 & 33.79 & 2.78 \\
\hline $\mathbf{3}$ & Days to first male Flower & 0.643 & 28.62 & 2.75 \\
\hline $\mathbf{4}$ & Node to first male flower & 0.076 & 1.488 & 0.004 \\
\hline $\mathbf{5}$ & Node to first female flower & 0.84 & 29.64 & 0.85 \\
\hline $\mathbf{6}$ & Days to 50\% flowering & 3.622 & 40.44 & 2.55 \\
\hline $\mathbf{7}$ & Days to first harvest & 0.218 & 33.07 & 2.94 \\
\hline $\mathbf{8}$ & Days to last harvest & 4.149 & 50.03 & 1.909 \\
\hline $\mathbf{9}$ & Fruit length (cm) & 1.28 & 118.69 & 1.034 \\
\hline $\mathbf{1 0}$ & Fruit diameter (mm) & 2.804 & 130.54 & 1.55 \\
\hline $\mathbf{1 1}$ & Rind thickness (mm) & 0.0154 & 1.465 & 0.0276 \\
\hline $\mathbf{1 2}$ & Flesh thickness (cm) & 0.0136 & 1.530 & 0.025 \\
\hline $\mathbf{1 3}$ & Number o fruits p plant & 1.182 & 10.90 & 0.50 \\
\hline $\mathbf{1 4}$ & Average fruit weight (g) & 6.85 & 67.21 & 1.96 \\
\hline $\mathbf{1 5}$ & Fruit yield per plant (kg) & 142.77 & 2083.24 & 55.21 \\
\hline $\mathbf{1 6}$ & Fruit yield/ ha (ton) & 0.084 & 0.829 & 0.024 \\
\hline $\mathbf{1 7}$ & TSS & 0.158 & 0.096 & 0.157 \\
\hline $\mathbf{1 8}$ & Vitamin C (m) & 0.77 & 0.255 & 0.232 \\
\hline
\end{tabular}

$*$ and ** indicate significant at $5 \%$ and $1 \%$ level, respectively. 
Table.3 Mean performance of eighteen genotypes of ridge gourd

\begin{tabular}{|c|c|c|c|c|c|c|c|c|c|}
\hline Genotypes & $\begin{array}{c}\text { Vine } \\
\text { Length } \\
\text { (cm at } 90 \\
\text { DAS }\end{array}$ & $\begin{array}{c}\text { Days to } \\
\text { first } \\
\text { female } \\
\text { flower }\end{array}$ & $\begin{array}{c}\text { Days to } \\
\text { first } \\
\text { male } \\
\text { Flower }\end{array}$ & $\begin{array}{l}\text { Node to } \\
\text { first male } \\
\text { flower }\end{array}$ & $\begin{array}{l}\text { Node to } \\
\text { first female } \\
\text { flower }\end{array}$ & $\begin{array}{c}\text { Days to } \\
50 \% \\
\text { flowering }\end{array}$ & $\begin{array}{c}\text { Days to } \\
\text { first } \\
\text { harvest }\end{array}$ & $\begin{array}{c}\text { Days to } \\
\text { last } \\
\text { harvest }\end{array}$ & $\begin{array}{l}\text { Fruit } \\
\text { length } \\
(\mathrm{cm})\end{array}$ \\
\hline 1 2018/RIGHYB - 1 & 319.64 & 41.02 & 36.11 & 3.26 & 9.72 & 44.04 & 51.28 & 82.64 & 23.03 \\
\hline 2 2018/RIGHYB - 2 & 297.8 & 42.94 & 38.31 & 3.33 & 12.15 & 45.89 & 56.02 & 85.95 & 17.55 \\
\hline 3 2018/RIGHYB - 3 & 305.2 & 44.93 & 40.42 & 4.45 & 15.01 & 48.13 & 56.69 & 86.28 & 27.68 \\
\hline 4 2018/RIGHYB - 4 & 326.1 & 39.8 & 34.89 & 3.04 & 7.87 & 42.45 & 49.88 & 79.78 & 27.99 \\
\hline 5 2018/RIGHYB - 5 & 233.07 & 48.94 & 44.56 & 5.15 & 18.9 & 50.48 & 59.37 & 89.51 & 21.71 \\
\hline 6 2018/RIGHYB - 6 & 239.69 & 45.87 & 41.36 & 4.61 & 15.97 & 49.78 & 57.43 & 90.67 & 29.08 \\
\hline 7 2018/RIGHYB - 7 & 219.69 & 47.57 & 43.12 & 4.2 & 12.68 & 50.39 & 56.44 & 88.53 & 24.6 \\
\hline 8 2017/RIGHYB - 1 & 278.01 & 48.82 & 43.78 & 5.19 & 17.48 & 51.91 & 59.21 & 91.75 & 30.65 \\
\hline 9 2017/RIGHYB - 2 & 284.54 & 47.06 & 42.9 & 4.7 & 17.15 & 50.77 & 58.4 & 90.46 & 31.75 \\
\hline 10 2017/RIGHYB - 5 & 241.98 & 49.36 & 45 & 4.28 & 14.73 & 53.48 & 60.38 & 94.21 & 14.53 \\
\hline 11 2017/RIGHYB - 6 & 290.8 & 48.75 & 43.76 & 4.25 & 13.2 & 52.34 & 60.62 & 92.2 & 17.93 \\
\hline 12 2017/RIGVAR - 1 & 270.08 & 46.87 & 47.42 & 4.31 & 13.83 & 53.97 & 60.22 & 93.46 & 16.3 \\
\hline 13 2017/RIGVAR - 2 & 207.48 & 49.43 & 44.65 & 4.29 & 16.26 & 51.77 & 59.52 & 89.2 & 19.11 \\
\hline 14 2017/RIGVAR - 3 & 236.3 & 47.88 & 43.44 & 4.94 & 17.02 & 52.31 & 58.67 & 88.61 & 21.12 \\
\hline 15 2017/RIGVAR - 4 & 280.55 & 48.18 & 43.72 & 4.32 & 14.65 & 51.8 & 59.55 & 91.43 & 16.02 \\
\hline 16 2017/RIGVAR - 5 & 267.27 & 46.22 & 46.86 & 4.39 & 16.38 & 54.55 & 62.15 & 93.26 & 16.44 \\
\hline 17 2017/RIGVAR - 6 & 257.97 & 49.4 & 45.31 & 4.43 & 17.24 & 52.72 & 60.04 & 92.32 & 17.12 \\
\hline 18 Rekha & 192.18 & 52.15 & 47.45 & 5.74 & 20.44 & 57.86 & 63.48 & 96.26 & 16.8 \\
\hline Mean & 263.7972 & 46.955 & 42.9478 & 4.3822 & 15.0378 & 50.8133 & 58.2972 & 89.8067 & 21.6339 \\
\hline C.V. & 2.5863 & 3.3511 & 3.7566 & 1.5874 & 6.0737 & 3.1265 & 3.1926 & 1.4277 & 4.139 \\
\hline S.E. & 3.9391 & 0.9085 & 0.9315 & 0.0402 & 0.5273 & 0.9172 & 1.0746 & 0.7402 & 0.517 \\
\hline C.D. 5\% & 11.321 & 2.6109 & 2.6771 & 0.1154 & 1.5155 & 2.6362 & 3.0883 & 2.1274 & 1.4858 \\
\hline Range Lowest & 192.18 & 39.8 & 34.89 & 3.04 & 7.87 & 42.45 & 49.88 & 79.78 & 14.53 \\
\hline Range Highest & 326.1 & 52.15 & 47.45 & 5.74 & 20.44 & 57.86 & 63.48 & 96.26 & 31.75 \\
\hline
\end{tabular}


Table.4 Mean performance of eighteen genotypes of ridge gourd

\begin{tabular}{|c|c|c|c|c|c|c|c|c|c|}
\hline Genotypes & $\begin{array}{c}\text { Fruit } \\
\text { diameter } \\
(\mathbf{m m})\end{array}$ & $\begin{array}{c}\text { Rind } \\
\text { thicknes } \\
\text { s (mm) }\end{array}$ & $\begin{array}{l}\text { Flesh } \\
\text { thickne } \\
\text { ss (cm) }\end{array}$ & $\begin{array}{c}\text { Number } \\
\text { ox fruit } \\
\text { per plant }\end{array}$ & $\begin{array}{c}\text { Average } \\
\text { fruit weight } \\
\text { (g) }\end{array}$ & $\begin{array}{c}\text { Fruit yield } \\
\text { per Plant } \\
(\mathrm{kg})\end{array}$ & $\begin{array}{c}\text { Fruit } \\
\text { yield/ ha } \\
\text { (ton) }\end{array}$ & TSS & $\begin{array}{c}\text { Vitamin } \\
\text { C (mg) }\end{array}$ \\
\hline 1 2018/RIGHYB - 1 & 43.370 & 3.560 & 3.630 & 13.240 & 140.050 & 1.850 & 16.650 & 4.060 & 3.100 \\
\hline 2 2018/RIGHYB - 2 & 42.860 & 4.600 & 4.650 & 13.970 & 126.510 & 1.770 & 15.930 & 3.940 & 4.070 \\
\hline 3 2018/RIGHYB - 3 & 38.710 & 3.190 & 3.140 & 14.210 & 143.160 & 1.990 & 17.910 & 3.980 & 3.880 \\
\hline 4 2018/RIGHYB - 4 & 45.360 & 3.370 & 3.570 & 13.660 & 154.630 & 2.120 & 19.080 & 3.890 & 4.350 \\
\hline 5 2018/RIGHYB - 5 & 41.120 & 3.250 & 3.190 & 12.990 & 116.190 & 1.510 & 13.590 & 4.330 & 4.090 \\
\hline 6 2018/RIGHYB - 6 & 39.170 & 3.040 & 2.920 & 14.010 & 133.470 & 1.870 & 16.830 & 4.050 & 4.050 \\
\hline 7 2018/RIGHYB - 7 & 53.850 & 4.830 & 4.830 & 13.140 & 164.040 & 2.150 & 19.350 & 4.150 & 4.170 \\
\hline 8 2017/RIGHYB - 1 & 47.510 & 3.380 & 3.350 & 12.280 & 152.140 & 1.860 & 16.740 & 3.810 & 4.020 \\
\hline 9 2017/RIGHYB - 2 & 59.780 & 4.140 & 4.030 & 14.950 & 182.820 & 2.720 & 24.480 & 4.070 & 4.010 \\
\hline 10 2017/RIGHYB - 5 & 48.550 & 5.140 & 5.100 & 12.520 & 160.480 & 2.000 & 18.000 & 4.200 & 4.260 \\
\hline 11 2017/RIGHYB - 6 & 44.490 & 3.240 & 3.260 & 13.450 & 147.310 & 1.970 & 17.730 & 4.100 & 4.370 \\
\hline 12 2017/RIGVAR - 1 & 45.370 & 3.440 & 3.710 & 10.480 & 116.690 & 1.210 & 10.890 & 3.870 & 4.480 \\
\hline 13 2017/RIGVAR - 2 & 48.780 & 4.040 & 4.140 & 9.600 & 117.430 & 1.100 & 9.900 & 3.690 & 4.170 \\
\hline 14 2017/RIGVAR - 3 & 36.940 & 2.630 & 2.580 & 12.170 & 98.540 & 1.190 & 10.710 & 4.070 & 4.010 \\
\hline 15 2017/RIGVAR - 4 & 43.680 & 3.450 & 3.690 & 10.640 & 105.130 & 1.110 & 9.990 & 4.160 & 4.280 \\
\hline 16 2017/RIGVAR - 5 & 41.620 & 3.540 & 3.300 & 11.310 & 107.320 & 1.210 & 10.890 & 4.120 & 4.090 \\
\hline 17 2017/RIGVAR - 6 & 40.380 & 3.350 & 3.310 & 10.740 & 111.640 & 1.200 & 10.800 & 3.790 & 4.150 \\
\hline 18 Rekha & 44.660 & 3.510 & 3.580 & 6.980 & 108.600 & 0.760 & 6.840 & 3.770 & 3.870 \\
\hline Mean & 44.789 & 3.650 & 3.666 & 12.241 & 132.564 & 1.644 & 14.795 & 4.003 & 4.079 \\
\hline C.V. & 2.973 & 2.540 & 5.040 & 5.252 & 2.538 & 5.432 & 5.432 & 12.105 & 12.073 \\
\hline S.E. & 0.769 & 0.054 & 0.107 & 0.371 & 1.943 & 0.052 & 0.464 & 0.280 & 0.284 \\
\hline C.D. 5\% & 2.210 & 0.154 & 0.307 & 1.067 & 5.584 & 0.148 & 1.334 & - & - \\
\hline Range Lowest & 36.940 & 2.630 & 2.580 & 6.980 & 98.540 & 0.760 & 6.840 & 3.690 & 3.100 \\
\hline Range Highest & 59.780 & 5.140 & 5.100 & 14.950 & 182.820 & 2.720 & 24.480 & 4.330 & 4.480 \\
\hline
\end{tabular}


Table.5 Estimation of genetic variability, GCV, PCV, Heritability, genetic advance and genetic advance as per cent of mean for 18 characters in ridge gourd genotypes

\begin{tabular}{|c|c|c|c|c|c|c|}
\hline Sl. No. & Character & $\begin{array}{c}\text { Genotypic } \\
\text { coefficient of } \\
\text { variation }\end{array}$ & $\begin{array}{l}\text { Phenotypic } \\
\text { coefficient of } \\
\text { variation }\end{array}$ & $\begin{array}{l}\text { Heritability in } \\
\text { broad sense }\left(h^{2} b\right)\end{array}$ & $\begin{array}{l}\text { Genetic } \\
\text { Advance }\end{array}$ & $\begin{array}{c}\text { Genetic } \\
\text { Advance as \% } \\
\text { of mean }\end{array}$ \\
\hline 1 & Vine Length $(\mathrm{cm})$ at 90 DAS & 13.57 & 13.97 & 94.90 & 72.22 & 27.24 \\
\hline 2 & Days to first female flower & 6.26 & 7.19 & 75.80 & 5.26 & 11.22 \\
\hline 3 & Days to first male Flower & 7.53 & 8.49 & 78.70 & 5.87 & 13.77 \\
\hline 4 & Node to first male flower & 15.783 & 15.854 & 99.100 & 1.442 & 32.36 \\
\hline 5 & Node to first female flower & 20.133 & 21.013 & 91.80 & 6.11 & 39.73 \\
\hline 6 & Days to $50 \%$ flowering & 7.020 & 7.699 & 83.20 & 6.67 & 13.188 \\
\hline 7 & Days to first harvest & 5.449 & 6.197 & 77.30 & 5.73 & 9.869 \\
\hline 8 & Days to last harvest & 4.459 & 4.717 & 89.400 & 7.80 & 8.68 \\
\hline 9 & Fruit length $(\mathrm{cm})$ & 27.177 & 27.533 & 97.400 & 12.73 & 55.260 \\
\hline 10 & Fruit diameter (mm) & 14.33 & 14.58 & 96.50 & 13.27 & 29.003 \\
\hline 11 & Rind thickness (mm) & 18.523 & 19.050 & 94.500 & 1.387 & 37.102 \\
\hline 12 & Flesh thickness (cm) & 19.006 & 19.475 & 95.200 & 1.424 & 38.211 \\
\hline 13 & Number o fruits p plant & 14.868 & 15.907 & 87.400 & 3.585 & 28.629 \\
\hline 14 & Average fruit weight (g) & 29.499 & 30.802 & 91.700 & 9.201 & 58.196 \\
\hline 15 & Fruit yield per plant (kg) & 18.802 & 19.554 & 92.400 & 51.498 & 37.240 \\
\hline 16 & Fruit yield/ ha (ton) & 29.499 & 30.802 & 91.700 & 1.002 & 58.196 \\
\hline 17 & TSS & 3.539 & 9.171 & 14.900 & 0.114 & 2.813 \\
\hline 18 & Vitamin C (mg) & 2.153 & 12.075 & 3.200 & 0.032 & 0.791 \\
\hline
\end{tabular}


Table.6 Genotypic correlation ( $\mathrm{rg}$ ) between yield and yield attributes for eighteen characters in Ridge Gourd genotypes

\begin{tabular}{|c|c|c|c|c|c|c|c|c|c|c|c|c|c|c|c|c|c|c|}
\hline & 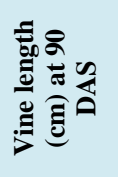 & 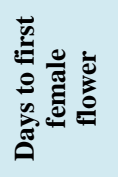 & 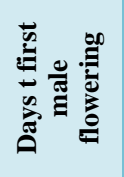 & 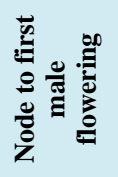 & 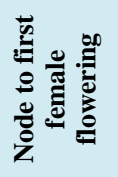 & 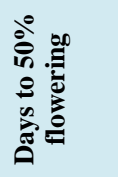 & 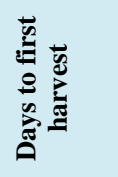 & 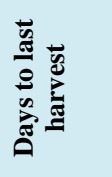 & 昰 & 离 & 急总 & 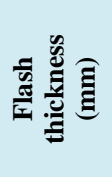 & 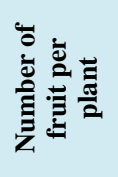 & $\mathscr{n}$ & 岂 & 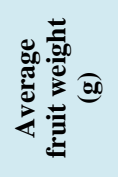 & 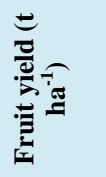 & 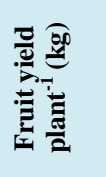 \\
\hline $\begin{array}{l}\text { Vine length } \\
(\mathrm{cm}) \text { at } 90 \\
\text { DAS }\end{array}$ & 1.00 & $0 . \overline{-}$ & $0.656^{* *}$ & $0.670 * *$ & $0.687^{-} * *$ & $0.672 * *$ & $0 . \overline{605^{* *}}$ & $-\overline{-}^{-}$ & $0.287^{*}$ & -0.08 & -0.15 & -0.10 & $0.583^{* *}$ & -0.24 & 0.23 & $0.436 * *$ & 0.07 & $0.275^{*}$ \\
\hline $\begin{array}{l}\text { Days to first } \\
\text { female flower }\end{array}$ & & 1.00 & $0.892 * *$ & $0.892 * *$ & $0.904 * *$ & $0.921 * *$ & $0.986^{* *}$ & $0.896^{* *}$ & $-0.329 *$ & 0.13 & -0.05 & -0.11 & $\begin{array}{c}- \\
0.597 * *\end{array}$ & 0.24 & -0.22 & $\overline{0 .}-\overline{23} * *$ & -0.18 & -0.26 \\
\hline $\begin{array}{l}\text { Days t first } \\
\text { male flowering }\end{array}$ & & & 1.00 & $0.796^{* *}$ & $0.791 * *$ & $0.993 * *$ & $0.852^{* *}$ & $0.971 * *$ & $-\overline{-}$ & 0.05 & -0.05 & -0.15 & $\stackrel{-}{0.691 * *}$ & $0.471 * *$ & -0.21 & $\stackrel{-}{-}$ & -0.11 & $-\overline{-}$ \\
\hline $\begin{array}{l}\text { Node to first } \\
\text { male flowering }\end{array}$ & & & & 1.00 & $0.956^{* *}$ & $0.820 * *$ & $0.867 * *$ & $0.792 * *$ & -0.02 & -0.05 & $0.295^{-}$ & $-\overline{-}$ & $\stackrel{-}{0.460 * *}$ & 0.04 & -0.07 & $\stackrel{-}{0.361 * *}$ & -0.07 & -0.26 \\
\hline $\begin{array}{l}\text { Node to first } \\
\text { female } \\
\text { flowering }\end{array}$ & & & & & 1.00 & $0.803 * *$ & $0.901 * *$ & $0.781 * *$ & -0.09 & -0.08 & -0.21 & $-0.334^{*}$ & $-\overline{-}$ & 0.21 & 0.07 & $\overline{-}-\overline{-} 4 * *$ & -0.07 & $0.367 * *$ \\
\hline $\begin{array}{l}\text { Days to } 50 \% \\
\text { flowering }\end{array}$ & & & & & & 1.00 & $0.845^{* *}$ & $0.962 * *$ & $\overline{0.515 * *}$ & 0.01 & -0.04 & -0.13 & $-\overline{-}$ & $0.410 * *$ & -0.25 & $0.571 * *$ & -0.07 & $0.398 * *$ \\
\hline $\begin{array}{c}\text { Days to first } \\
\text { harvest }\end{array}$ & & & & & & & 1.00 & $0.854^{* *}$ & $\stackrel{-}{0.503^{* *}}$ & -0.05 & -0.09 & -0.18 & $\stackrel{-}{0.640 * *}$ & $0.392 * *$ & $0.551 * *$ & $\overline{-}-\overline{12 * *}$ & -0.07 & $0.375^{* *}$ \\
\hline $\begin{array}{l}\text { Days to last } \\
\text { harvest }\end{array}$ & & & & & & & & 1.00 & $\overline{-}^{-}{ }^{*} 6^{* *}$ & 0.08 & 0.06 & 0.01 & $\stackrel{-}{0.559 * *}$ & 0.21 & $\stackrel{-}{0.557 * *}$ & $\stackrel{-}{-} 0.377^{* *}$ & -0.04 & -0.22 \\
\hline $\begin{array}{c}\text { Fruit length } \\
(\mathrm{cm})\end{array}$ & & & & & & & & & 1.00 & $0.295^{*}$ & -0.17 & -0.15 & $0.636^{* *}$ & $0 . \overline{538^{* *}}$ & 0.26 & $0.678 * *$ & 0.15 & $0.598 * *$ \\
\hline $\begin{array}{c}\text { Fruit } \\
\text { diameter }(\mathbf{c m})\end{array}$ & & & & & & & & & & 1.00 & $\begin{array}{c}0.655^{*} \\
*\end{array}$ & $0.700 * *$ & 0.16 & -0.09 & -0.12 & $0.569 * *$ & 0.07 & $0.741 * *$ \\
\hline $\begin{array}{l}\text { Rind thickness } \\
\text { (mm) }\end{array}$ & & & & & & & & & & & 1.00 & 1.00 & 0.13 & -0.10 & -0.21 & $0.382 * *$ & -0.02 & $0.514 * *$ \\
\hline $\begin{array}{c}\begin{array}{c}\text { Flash } \\
\text { thickness } \\
(\mathbf{m m})\end{array}\end{array}$ & & & & & & & & & & & & 1.00 & 0.11 & -0.15 & -0.26 & $0.381 * *$ & 0.06 & $0.523 * *$ \\
\hline $\begin{array}{l}\text { Number of } \\
\text { fruit per plant }\end{array}$ & & & & & & & & & & & & & 1.00 & $-\overline{-}$ & -0.03 & $0.871 * *$ & $\begin{array}{c}0.393 * \\
*\end{array}$ & $0.666^{* *}$ \\
\hline TSS & & & & & & & & & & & & & & 1.00 & 0.05 & $-\overline{-}$ & -0.12 & $0.621 * *$ \\
\hline Vitamin C & & & & & & & & & & & & & & & 1.00 & -0.08 & -0.01 & -0.13 \\
\hline $\begin{array}{l}\text { Average fruit } \\
\text { weight (g) }\end{array}$ & & & & & & & & & & & & & & & & 1.00 & 0.23 & $0.944 * *$ \\
\hline $\begin{array}{c}\text { Fruit yield (t } \\
\left.\text { ha }^{-1}\right)\end{array}$ & & & & & & & & & & & & & & & & & 1.00 & 0.07 \\
\hline $\begin{array}{l}\text { Fruit yield } \\
\text { plant }^{-1}(\mathbf{k g})\end{array}$ & & & & & & & & & & & & & & & & & & 1.00 \\
\hline
\end{tabular}


Table.7 Phenotypic correlation (rp) between yield and yield attributes for eighteen characters in Ridged gourd genotypes

\begin{tabular}{|c|c|c|c|c|c|c|c|c|c|c|c|c|c|c|c|c|c|c|}
\hline & 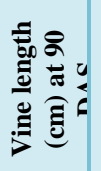 & 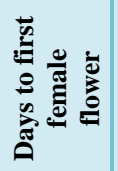 & 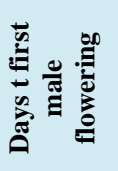 & 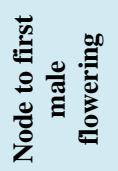 & 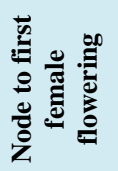 & 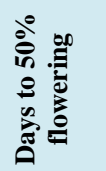 & 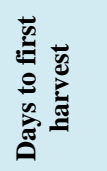 & 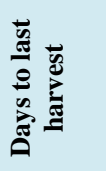 & 氞 & 离 & 氞产总 & 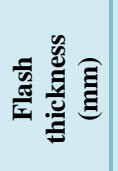 & 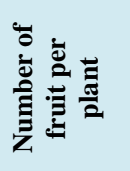 & $\mathscr{\omega}$ & U & 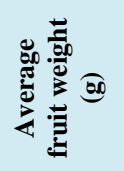 & 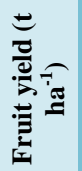 & 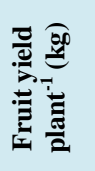 \\
\hline $\begin{array}{l}\text { Vine length }(\mathrm{cm}) \\
\text { at } 90 \text { DAS }\end{array}$ & 1.00 & $\begin{array}{c}-0.6907 \\
* * *\end{array}$ & $\begin{array}{c}-0.5823 \\
* * *\end{array}$ & $\begin{array}{c}-0.6480 \\
* * *\end{array}$ & $\begin{array}{l}-0.6147 \\
* * *\end{array}$ & $\begin{array}{c}-0.5798 \\
* * *\end{array}$ & $\begin{array}{c}-0.5545 \\
* * *\end{array}$ & $\begin{array}{c}-0.5546 \\
* * *\end{array}$ & 0.27 & -0.07 & -0.16 & -0.11 & $0.5245 * *$ & -0.20 & $\overline{-}$ & $0.4192 *$ & 0.02 & $0.270^{*}$ \\
\hline $\begin{array}{l}\text { Days to first } \\
\text { female flower }\end{array}$ & & 1.00 & $\begin{array}{c}0.8604 \\
* * *\end{array}$ & $\begin{array}{l}0.7990 \\
* * *\end{array}$ & $\begin{array}{l}0.7671 \\
* * *\end{array}$ & $\begin{array}{c}0.8187 \\
* * *\end{array}$ & $\begin{array}{l}0.8465 \\
* * *\end{array}$ & $\begin{array}{c}0.8047 \\
* * *\end{array}$ & -0.32 & 0.13 & -0.03 & -0.09 & $\begin{array}{c}-0.5406 \\
* * *\end{array}$ & 0.24 & 0.09 & $\begin{array}{c}-0.3917 \\
*\end{array}$ & $\overline{-}$ & -0.23 \\
\hline $\begin{array}{l}\text { Days t first male } \\
\text { flowering }\end{array}$ & & & 1.00 & $\begin{array}{c}0.7345 \\
* * *\end{array}$ & $\begin{array}{c}0.7406 \\
* * *\end{array}$ & $\begin{array}{l}0.9221 \\
* * *\end{array}$ & $\begin{array}{l}0.8866 \\
* * *\end{array}$ & $\begin{array}{c}0.8300 \\
* * *\end{array}$ & $\begin{array}{c}-0.4819 \\
* *\end{array}$ & 0.03 & -0.05 & -0.10 & $\begin{array}{l}-0.5853 \\
* * *\end{array}$ & $\begin{array}{l}0.4111 \\
*\end{array}$ & 0.19 & $\begin{array}{c}-0.5039 \\
* *\end{array}$ & $\begin{array}{c}- \\
0.19\end{array}$ & $\begin{array}{c}- \\
0.368^{*} \\
*\end{array}$ \\
\hline $\begin{array}{l}\text { Node to first } \\
\text { male flowering }\end{array}$ & & & & 1.00 & $\begin{array}{l}0.9136 \\
* * *\end{array}$ & $\begin{array}{l}0.7571 \\
* * *\end{array}$ & $\begin{array}{l}0.7525 \\
* * *\end{array}$ & $\begin{array}{l}0.7413 \\
* * *\end{array}$ & -0.02 & -0.05 & -0.29 & $\begin{array}{c}-0.3386 \\
*\end{array}$ & $\begin{array}{c}-0.4289 \\
* *\end{array}$ & 0.05 & 0.07 & $\begin{array}{c}-0.3538 \\
*\end{array}$ & $-\overline{0}$ & -0.26 \\
\hline $\begin{array}{l}\text { Node to first } \\
\text { female flowering }\end{array}$ & & & & & 1.00 & $\begin{array}{l}0.7605 \\
* * *\end{array}$ & $\begin{array}{c}0.7496 \\
* * *\end{array}$ & $\begin{array}{c}0.6755 \\
* * *\end{array}$ & -0.10 & -0.08 & -0.22 & -0.29 & $-0.3830 *$ & 0.17 & 0.06 & $\begin{array}{c}-0.3798 \\
*\end{array}$ & $\begin{array}{c}- \\
0.08\end{array}$ & $-0.341^{*}$ \\
\hline $\begin{array}{c}\text { Days to 50\% } \\
\text { flowering }\end{array}$ & & & & & & 1.00 & $\begin{array}{l}0.8840 \\
* * *\end{array}$ & $\begin{array}{l}0.8912 \\
* * *\end{array}$ & $\begin{array}{c}-0.4725 \\
* *\end{array}$ & 0.02 & -0.06 & -0.13 & $\begin{array}{l}-0.6008 \\
* * *\end{array}$ & 0.33 & 0.11 & $\begin{array}{l}-0.5050 \\
* *\end{array}$ & $-\overline{-}$ & $\begin{array}{c}- \\
0.365^{*} \\
*\end{array}$ \\
\hline $\begin{array}{l}\text { Days to first } \\
\text { harvest }\end{array}$ & & & & & & & 1.00 & $\begin{array}{l}0.8403 \\
* * *\end{array}$ & $\begin{array}{c}-0.4362 \\
* *\end{array}$ & 0.00 & -0.08 & -0.16 & $\begin{array}{c}-0.5027 \\
* *\end{array}$ & 0.19 & $\overline{0}-$ & $\begin{array}{c}-0.4372 \\
* *\end{array}$ & $\overline{0}-15$ & $-0.329 *$ \\
\hline $\begin{array}{c}\text { Days to last } \\
\text { harvest }\end{array}$ & & & & & & & & 1.00 & $\begin{array}{c}-0.4158 \\
*\end{array}$ & 0.10 & 0.06 & -0.01 & $\begin{array}{c}-0.4878 \\
* *\end{array}$ & 0.18 & 0.11 & $\begin{array}{c}-0.3488 \\
*\end{array}$ & 0.03 & -0.20 \\
\hline Fruit length (cm) & & & & & & & & & 1.00 & 0.27 & -0.18 & -0.16 & $\begin{array}{c}0.5974 \\
* * *\end{array}$ & $\begin{array}{c}-0.5166 \\
* *\end{array}$ & $\begin{array}{c}- \\
0.13\end{array}$ & $\begin{array}{c}0.6567 \\
* * *\end{array}$ & 0.18 & $\begin{array}{c}0.573 * \\
*\end{array}$ \\
\hline $\begin{array}{l}\text { Fruit diameter } \\
\text { (cm) }\end{array}$ & & & & & & & & & & 1.00 & $\begin{array}{c}0.6315 \\
* * *\end{array}$ & $\begin{array}{c}0.6265 \\
* * *\end{array}$ & 0.15 & -0.09 & 0.00 & $\begin{array}{l}0.5397 \\
* * *\end{array}$ & 0.03 & $\begin{array}{c}0.709 * \\
*\end{array}$ \\
\hline $\begin{array}{l}\text { Rind thickness } \\
\text { (mm) }\end{array}$ & & & & & & & & & & & 1.00 & $\begin{array}{c}0.9650 \\
* * *\end{array}$ & 0.11 & -0.04 & 0.08 & $0.3673 *$ & 0.01 & $\begin{array}{c}0.509 * \\
*\end{array}$ \\
\hline $\begin{array}{c}\text { Flash thickness } \\
(\mathbf{m m})\end{array}$ & & & & & & & & & & & & 1.00 & 0.08 & -0.03 & 0.10 & $0.3523 *$ & $\overline{-}$ & $\begin{array}{c}0.504 * \\
*\end{array}$ \\
\hline $\begin{array}{c}\text { Number of fruit } \\
\text { per plant }\end{array}$ & & & & & & & & & & & & & 1.00 & $\begin{array}{c}-0.5650 \\
* * *\end{array}$ & - & $\begin{array}{c}0.8599 \\
* * *\end{array}$ & 0.24 & $\begin{array}{l}0.607 * \\
*\end{array}$ \\
\hline TSS & & & & & & & & & & & & & & 1.00 & 0.01 & $\begin{array}{c}-0.5980 \\
* * *\end{array}$ & $\overline{-}$ & $\begin{array}{c}- \\
0.514 * \\
*\end{array}$ \\
\hline Vitamin C & & & & & & & & & & & & & & & 1.00 & 0.02 & 0.08 & 0.04 \\
\hline $\begin{array}{c}\text { Average fruit } \\
\text { weight (g) }\end{array}$ & & & & & & & & & & & & & & & & 1.00 & 0.15 & $\begin{array}{l}0.924 * \\
*\end{array}$ \\
\hline Fruit yield $\left(\mathrm{t} \mathrm{ha}^{-1}\right)$ & & & & & & & & & & & & & & & & & 1.00 & 0.04 \\
\hline $\begin{array}{c}\text { Fruit yield plant }^{-1} \\
(\mathrm{~kg})\end{array}$ & & & & & & & & & & & & & & & & & & 1.00 \\
\hline
\end{tabular}


The genotype 2017/RIGHYB-5 was recorded high Fruit yield $(2.72 \mathrm{~kg} /$ plant and 24.48 ton/ha), whereas minimum Fruit yield per plant and per hectare was observed in genotype Rekha with $(0.76 \mathrm{~kg} / \mathrm{plant}$ and 6.84 ton/ha).

The study on genotypic and phenotypic coefficient of variation revealed that Higher magnitude of genotypic coefficient of variance (GCV) was recorded for Fruit yield/ ha (q) (29.499), Average fruit weight (g) (9.499) and Fruit length $(\mathrm{cm})(27.177)$ Node to first female flower (20.133). Higher magnitude of phenotypic coefficient of variance (PCV) was recorded for Fruit yield/ ha (q) (30.083), Average fruit weight (g) (30.083) and Fruit length $(\mathrm{cm})(27.533)$ Node to first female flower (20.013). The present findings are in accordance with the findings of Nath et al., (2009).

The heritability estimate were found to be high (>60) for almost all the characters viz., Vine Length $(\mathrm{cm})$ at 90 DAS (94.90), Days to first female flower (75.80), Days to first male Flower (78.70), Node to first male flower (99.100), Node to first female flower (91.80), Days to $50 \%$ flowering (83.20), Days to first harvest (77.30), Days to last harvest (89.400), Fruit length $(\mathrm{cm})$ (97.400), Fruit diameter (mm) (96.50), Rind thickness (mm) (94.500), Flesh thickness $(\mathrm{cm})$ (95.200), Number o fruits $\mathrm{p}$ plant (87.400), Average fruit weight (g) (91.700), Fruit yield per plant (kg) (92.400) and Fruit yield/ ha (ton) (91.700).

The estimation of genetic advance for all the characters are presented in Genetic advance as percent mean was categorized as low (0-10\%), moderate $(10-20 \%$ and $(\geq 20 \%)$ as given by Johnson et al., (1955) and Falconer and Mackay (1996). Genetic advance as mean was highest for Vine Length $(\mathrm{cm})$ at 90 DAS (72.22) and Fruit yield per plant (kg) (51.498). Genetic advance as per cent of mean was highest for Vine Length $(\mathrm{cm})$ at 90 DAS (27.24), Node to first male flower (32.36), Node to first female flower (39.73), Fruit length $(\mathrm{cm})(55.260)$, Fruit diameter (mm) (29.003), Rind thickness (mm)
(37.102), Flesh thickness (cm) (38.211), Number o fruits p plant (28.629), Average fruit weight (g) (58.196), Fruit yield per plant (kg) (37.240) and Fruit yield/ ha (ton) (58.196)

Genotypic correlation coefficient analysis revealed that fruit yield plant $^{-1}(\mathrm{~kg})$ showed positive significant association with Fruit length (cm) $(0.598 * *)$, Fruit diameter $(\mathrm{cm})(0.741 * *)$, Rind thickness $(\mathrm{mm})\left(0.514^{* *}\right)$, Flash thickness (mm) $\left(0.523^{* *}\right)$, Number of fruit per plant $\left(0.666^{* *}\right)$, Vine length $(\mathrm{cm})$ at 90 DAS $\left(0.275^{*}\right)$ and Average fruit weight $(\mathrm{g})(0.944 * *)$. While as negative significant association was observed with Days t first male flowering $(-0.407 * *)$, Node to first female flowering $(-0.367 * *)$, Days to $50 \%$ flowering $\left(-0.398^{* *}\right)$, Days to first harvest $\left(-0.375^{* *}\right)$ and TSS $\left(-0.621^{* *}\right)$

Phenotypic correlation coefficient analysis revealed that fruit yield plant $^{-1}(\mathrm{~kg})$ showed positive significant association with Fruit length (cm) $(0.573 * *)$, Fruit diameter $(\mathrm{cm})(0.709 * *)$, Rind thickness $(\mathrm{mm})\left(0.509^{* *}\right)$, Flash thickness (mm) $\left(0.504^{* *}\right)$, Number of fruit per plant $(0.607 * *)$, Average fruit weight $(\mathrm{g})(0.924 * *)$ and Vine length $(\mathrm{cm})$ at 90 DAS $\left(0.270^{*}\right)$. While as negative significant association was observed with Days t first male flowering ($\left.0.368^{* *}\right)$, Node to first female flowering ($\left.0.341^{*}\right)$, Days to $50 \%$ flowering $\left(-0.365^{* *}\right)$, Days to first harvest $\left(-0.329^{*}\right)$ and TSS ($0.514 * *)$.

On the basis of mean performance of eighteen genotypes of Ridge Gourd, genotype, 2017/RIGHYB-5 (2.72 kg/plant and 24.48 ton/ha) was found superior in terms of Fruit yield/ha (ton). On the basis of Analysis of variance significant difference was recorded for all the quantitative and qualitative traits indicating presence of large amount of variability in the genotypes.

Hence it can be concluded that Ridge GourdGenotype2017/RIGHYB-5 having highest yield can be utilized further for crop improvement programs. 


\section{References}

Ananthan M, Moorthy BG and Natarajan S. (2005) Genetic variability in ridge gourd (Luffa acutangula (L.) Roxb.), South Indian Horticulture, 53(1-6): 326-328.

Bharathi LK, Naik G and Dora DK. (2006) Correlation and path analysis in spine gourd (Momordica dioca Roxb.), The Orissa Journal Horticulture, 33(2): 105108.

Choudhary BR, Pandey S, Singh PK and Pandey V. (2014) Genetic diversity analysis for quantitative traits in hermaphrodite ridge gourd [Luffaa cutangula (Roxb.) L.], Indian Journal Horticulture, 71(2): 284-287

Devmore JP, Dhonukshe BL, Thaware BL, Bendale VW, Jadhav BB and Thprat TN. (2010) Genetic variability and heritability studies in bitter gourd (Momordica charantia L.), Journal Maharashtra Agriculture University, 35(1): 163-165.

Emina M, Berenji J, Ognjanov V, Mirjana L and Jelena C. (2012) Genetic variability of bottle gourd [Lagenaria siceraria (Mol.) standl] and its morphological characterization by multivariate analysis, Archives of Biological Science, Belgrade, 64 (2): 573-583.

Hanumegowda K, ShirolAM, Mulge R, Shantappa T and Prasadkumar. (2011) Genetic variability, heritability and genetic advance for yield and yield contributing characters in ridge gourd (Luffa acutangula (L.)Roxb), Journal of Asian Horticulture, 7 (4): 196-200.

Iqbal M A. (2009) Genetic Variability and PathCoefficient Analysis of Bitter Gourd (Momordica charantia L.), International Journal of Sustainable Agriculture,1(3): 53-57.

Kumar R, Ameta KD, Dubey RB and Sunil P.
(2013) Genetic variability, correlation and path analysis in sponge gourd (Luffa cylindrica Roem.), African Journal of Biotechnology, 12 (6): 539-543.

Mathew A, Markose LS, Rajan S and Devi SN. (2001) Genetic divergence in bottle gourd, Vegetable Science, 28(2): 121-123.

Nath, Vishwa., Lal, H., Rai, M., Rai, N. and Ram, D. (2009). Hierarchical Clustering and Character Association Studies in Cowpea [Vigna unguiculata (L.)Walp.]. Indian Journal of Plant Genetic Resources., 22: 22-25.

Peter KV. (1975) Genetic analysis of certain quantitative characters in tomato (Lycopersicon esculentum Mill.)Ph.D. Thesis, Govind Ballabh Pant University of Agriculture and Technology, Pantnagar.

Rao CR. (1952) Advanced Statistical Methods in Biometrical Research, John Willey and Sons, New York, pp. 357-359.

Singh AK, Pan RS and Bhavana P. (2013) Correlation and path coefficient analysis for quantitative traits in early season bottle gourd (Lagenaria siceraria(Mol.) Standl.), Vegetable science, 40 (2): 207209.

Varghese D, Chandramony and Seeja G. (2005) Correlation and path co-efficient analysis in ivy gourd, South Indian journal of Horticulture, 53(1-6): 120-125.

Wani KP, Ahmed N, Hussain K, Habib M and Kant RH. (2008) Correlation and path coefficient analysis in bottle gourd (Lagenaria siceraria L.) under temperate conditions of Kashmir valley, Environmental Ecology, 26(2A): 822824.

Yadav R and Ram HH. (2002) Correlation and path coefficient analysis in muskmelon, Haryana Journal Horticulture Science, 31 (1\&2): 74-76.

\section{How to cite this article:}

Akhila, K. and Devi Singh. 2020. Genetic Variability in Ridge Gourd (Luffa acuntangula (L.) Roxb.). Int.J.Curr.Microbiol.App.Sci. 9(10): 2774-2783. doi: https://doi.org/10.20546/ijcmas.2020.910.334 